\title{
A Microscopic Analysis of Crowd Behaviour at Dewan Sultan Ibrahim (DSI), UTHM on Convocation Day during the Emergency Situation by using the Crowd Behavior Simulator for Disaster Evacuation (CBS-DE) Method
}

\author{
Muhammad Salleh Abustan ${ }^{1, *}$, Mohd Firdaus Mohamad Ali ${ }^{1}$, Siti Hidayah Abu \\ Talib $^{1}$, Mohd Shalahuddin Adnan ${ }^{1}$
} ${ }^{1}$ Department of Infrastructure and Geomatic Engineering, Faculty of Civil Engineering and Environmental
Universiti Tun Hussein Onn Malaysia, 86400 Parit Raja, Batu Pahat, Johor.

Received 01 January 2018; accepted 15 April 2018, available online 07 May 2018

\begin{abstract}
This research focuses on the demonstration of the evacuation simulation as if emergency situation is occurred at Dewan Sultan Ibrahim (DSI) of Universiti Tun Hussein Onn Malaysia (UTHM) from the perspective of microscopic dynamics of crowd behaviour. The model was performed by using the DEM-based crowd behaviour simulator, namely as Crowd Behavior Simulator for Disaster Evacuation (CBS-DE) and its analysis is capable to track the trajectory and rotation of each individual. This research is based on two different cases in which the first case was using the average velocity of $1.16 \mathrm{~m} / \mathrm{s}$ for both male and female and the for the second case, the velocity of $1.25 \mathrm{~m} / \mathrm{s}$ was applied for male while lower velocity was applied for female which is $1.12 \mathrm{~m} / \mathrm{s}$. DSI building is considered as a well-designed building in terms of safety and convenience factors. This is due to the short time taken for the people to evacuate from the inside of the building by considering the crowds are familiar with the building as all the doors are easy to be seen.
\end{abstract}

Keywords: Dewan Sultan Ibrahim (DSI), Distinct-Element-Method (DEM), Crowd Behavior Simulator for Disaster Evacuation (CBS-DE), UTHM, FKAAS, Evacuation

\section{Introduction}

Pedestrian crowd behaviour study is beneficial for the purpose in designing and planning of a building because it helps to obtain the optimum safety of crowd inside the building principally during emergency cases such as fire and earthquake. As the number of high-rise superstructure keeps on increasing from year to year, this study has become crucial in gaining better understanding about crowd behaviour especially since the "911" incident $[3,10]$. In this study, the method used was microscopic modelling. According to available literatures, microscopic model is more accurate in predicting the crowd dynamics [1]. However, as a result of the high level of detail, microscopic simulation models tend to have long computation times [2]. The advantage of using microscopic approach is because the situation works like in the reality of occurrence where the crowd by individual behaviour and their motion characteristics can be created. The types of interaction can be classified as between human and human interaction, and also human and the environment interaction. These kinds of interactions are suitable in designing the evacuation details and the process. The evacuation process can be conducted in various scenarios that can be derived from a microscopic model named Crowd Behavior Simulator for Disaster Evacuation (CBS-DE) modelling. Hence, better planning can be produced from this model as prediction and derivation of different types of cases when doing the modelling. A realistic evacuation situation can be seen as the analysis will be presented in 3D virtual by using the Autodesk Maya software inside Dewan Sultan Ibrahim (DSI) where it is mainly functioned as a place for huge ceremonies or programmes that will be held by UTHM such as convocation.

\section{Human Behaviour}

Before creating a simulation model of the human movement accordingly, it is crucial to comprehend how the individuals behave during the evacuation situation and the simulator can only be ran if the dynamic of crowd behaviour occur. Human behaviour is based on individual's decision [7]. The survival aspect in human behaviour is the actions that people take based upon their perception of the situation, their intention to act, and the considerations involved before these actions are carried out [1]. In evacuation situation that caused by fires, the main reason to put into consideration is either the victims are having too much or too little information and 
instructions coming at that time. The situation can be seen when there are several people in the same room taking different decisions and shouting different information about blocked rooms, complex and dynamically changing situations that result in uncertainty, and time pressure.

\section{Evacuation Modelling}

This section describes the existing model of individual evacuation. In the evacuation simulation, there are two types of analysis and they are macroscopic and microscopic, where macroscopic is concerned with the collective behaviour of the evacuation process and that would be appropriate to be used to initial estimate of evacuation time. While, microscopic proves detailed interaction between each particles and flexible study relating to the crowd behaviour, bottleneck and at the same time the relationship between desired velocities of people can be conducted. The effect of panic and bottleneck situation may cause to serious injury and also death during the evacuation process. Therefore, the evacuation process inside DSI can be seen in Figure 1 to Figure 5 as how the crowds move from their initial positions to the exits where bottlenecks occur during the scene.

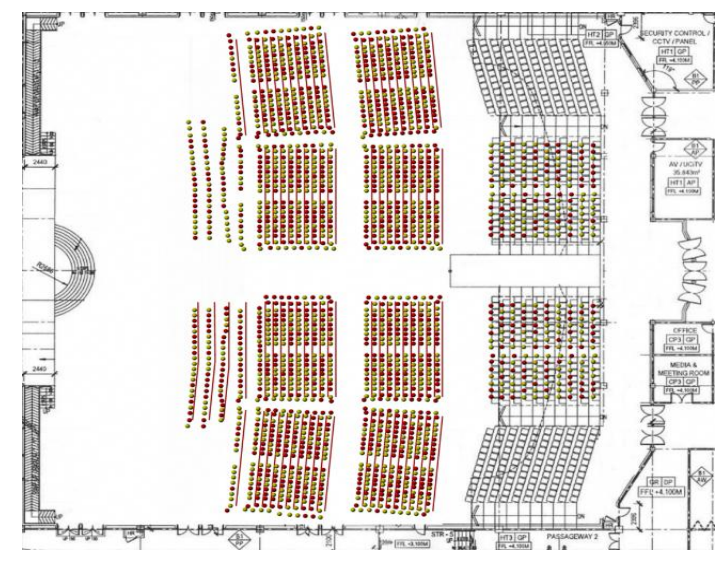

Fig. 1 Initial positions of people (red and yellow dots) inside DSI during the convocation ceremony.

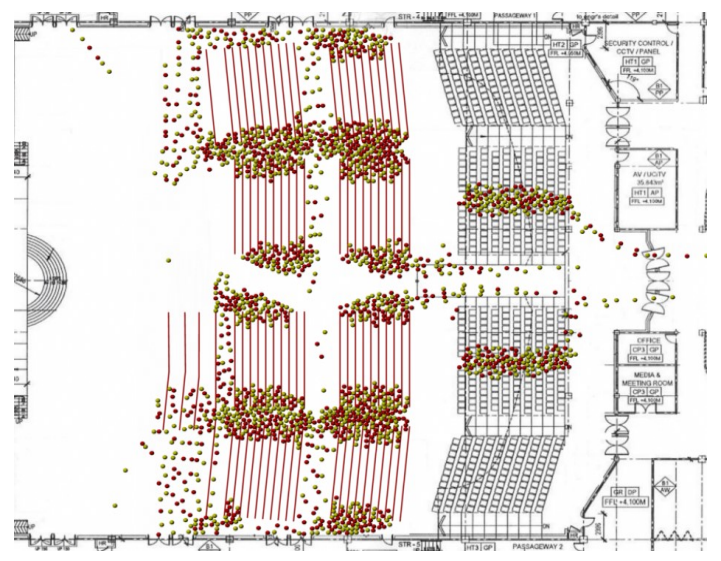

Fig. 2 This picture shows the evacuation process at 10 seconds where the crowds are heading towards the nearest exits.

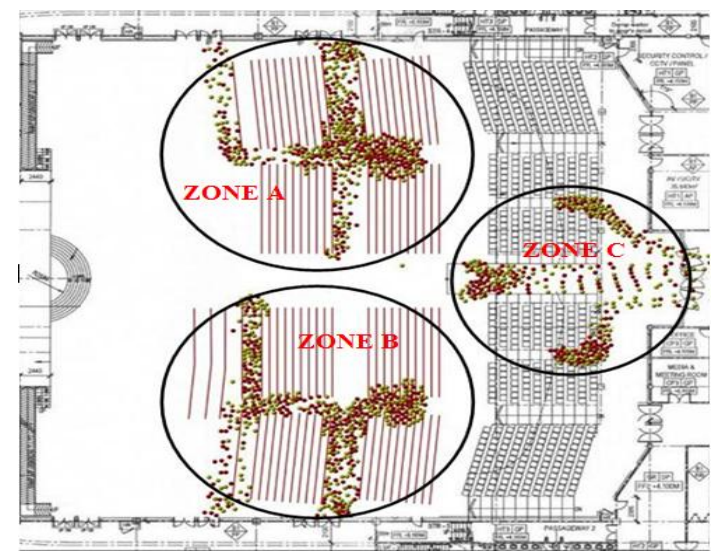

Fig. 3 People are gathering in three (3) different zones where the nearest exits are located.

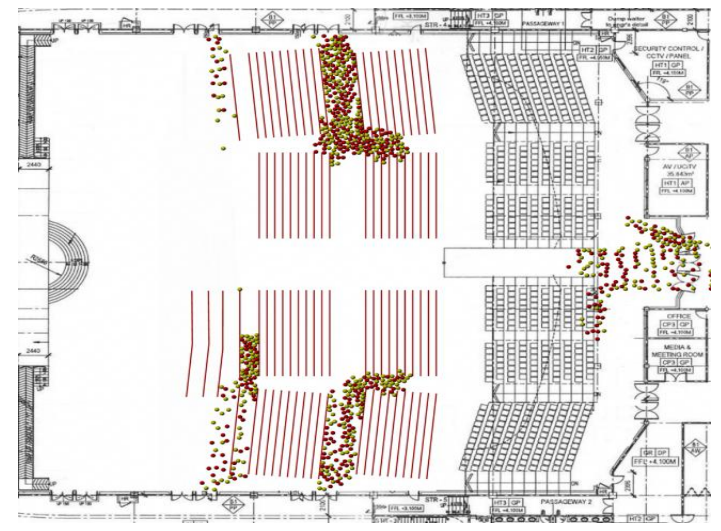

Fig. 4 About $50 \%$ of the people were evacuated at seconds 70 .

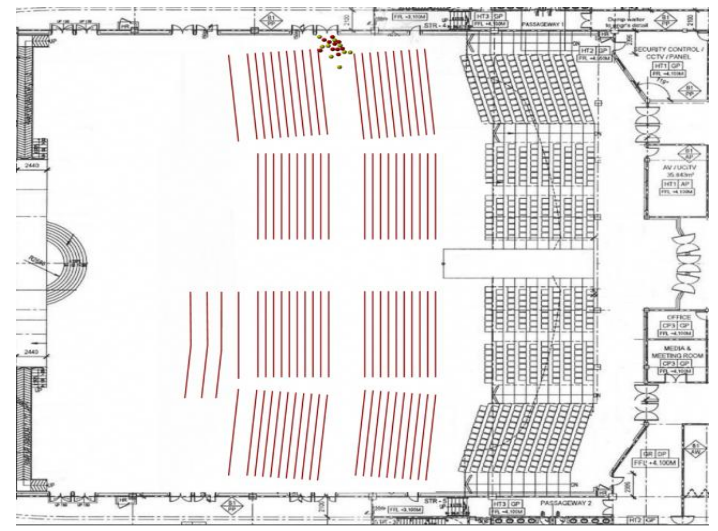

Fig. 5 It can be seen that a total evacuation was done after 140 seconds as shown in this figure.

Many of the existing evacuation models are using the individual perspective of occupants. However, this is not 
sufficient as the necessary checking whether the selected evacuation model has the capability to represent the observed evacuation behaviour of occupants or the other way around. A computer model that attempts to stimulate evacuation behaviour must be able to predict two things: the occupant actions performed (simulated) and the time taken to perform the action. In most cases, the improvement of current evacuation models is restricted to the developers as most of the software is closed-source that, the end-users cannot access the source code. According to [9], the flexibility and long-term capabilities in evacuation models is important for future improvements in addressing new emergency scenarios, procedural evacuations, interaction of the technology and model, real time manipulation, etc.

\section{CBS-DE Method}

According to [1], CBS-DE was first developed by Gotoh et al. in 2004 in order to evaluate the Tsunami evacuation situation. Person element motion is calculated through interactions with neighbouring elements and according to Newton's law of motion. Each person element is governed by translation and rotation equations of motion. Hence the motion of a person in CBS-DE [4] is shown as follow:

$$
\begin{aligned}
& m h i=\frac{1}{4} \varepsilon_{h i} \sigma_{h i} \beta_{h i} \pi d^{2} h i \\
& \operatorname{lhi}=\frac{1}{32} \varepsilon_{h i} \sigma_{h i} \beta_{h i} \pi d^{4} h i
\end{aligned}
$$

Where, $\varepsilon_{\text {hi }}$ is the volume coefficient concerning to the volume difference between the cylindrical element and the actual person, $\sigma_{\mathrm{hi}}$ is the density of the person $i, B_{\mathrm{hi}}$ is the body height of the person $i$, and $\mathrm{d}_{\mathrm{hi}}$ is the diameter of the person $i$. In this model, $\varepsilon_{h i}$ used is 0.31 by considering $m_{h i}=56.5 \mathrm{~kg}, \sigma_{h i}=980 \mathrm{~kg} / \mathrm{m}^{3}, B_{h i}=164.7 \mathrm{~cm}$ and $d_{h i}=$ $0.379 \mathrm{~m}$

\section{Representation of Perception Domain}

Vision is the main source information in controlling person's motion. In CBS-DE the person's $i$ vision is represented by a perception domain that reflects a person's space. The perception domain that illustrates the personal space of person $i$ as shown as in Figure 6 and it is used to evaluate interactions of person $i$ to adjacent person and to wall boundary. According to [4], psychologically people tend to keep a constant distance with each other when walking. CBS-DE assumes that a person unlikely notices anyone behind them and pay less attention to what occur then. For this reason, the vision field of person $i$, named angle vision, $\theta$ is introduced in the range of $[-60,60]$ from the left to the right of eye sight with respect to moving direction of person $\mathrm{i}$.

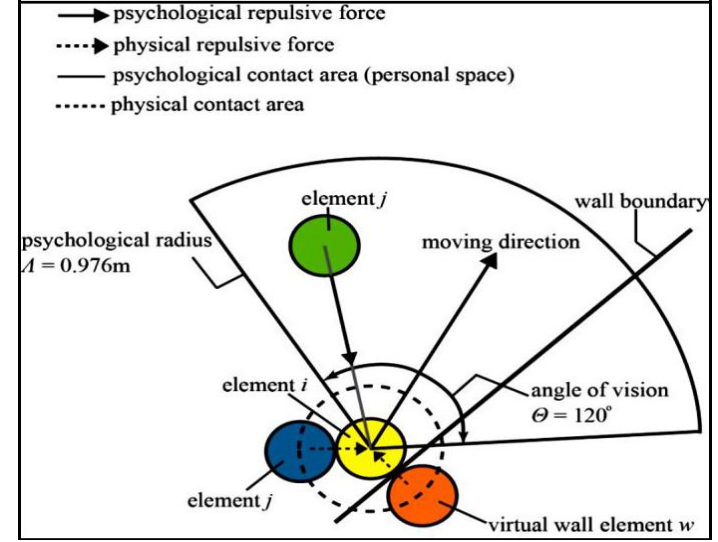

Fig. 6 Schematic diagram of the perception domain of a person, $i$.

\section{Inter-element Force}

The physical and psychological repulsive forces between person $i$ and $j$ are initiated if the relative distance between centroids of the person $i$ and $j$ satisfies as follow:

$$
\begin{aligned}
& \left|r_{i}-r_{j}\right| \leq \frac{d_{h i}-d_{h j}}{2} \\
& \left|r_{i}-r_{j}\right| \leq \Lambda
\end{aligned}
$$

Meanwhile, the physical repulsive force between person $i$ and virtual wall element $W$ acts when $\left|r_{i}-r_{w}\right| \leq \frac{d_{h i}-d_{w}}{2}$, where $\mathrm{W}$ is positioned on the wall having tangential contact with the person $i, r_{i}$ is the positional vector of the person $i, r_{w}$ is the positional vector of wall element $\mathrm{W}$, and $d_{w}$ is the diameter of the virtual wall element $\mathrm{W}$ with the same diameter as a person element (0.379). The total interacting force acting on the person $i$ is described as follows:

$$
\begin{aligned}
F_{i n h i} & =F_{i j}+F_{i W}+F_{p s i j} \\
F_{i j} & =\sum_{f(\neq 1)} f_{i j} ; F_{i W}=\sum w f_{i w} ; F_{p s i j}=\sum_{j(\neq 1)} f_{p s i j}
\end{aligned}
$$

$$
T_{h i}=\frac{1}{2}\left\{\sum_{j(\neq 1)}\left(r_{i}-r_{j}\right) X f_{i j}+\sum w\left(r_{w}-r_{i}\right) X f_{i W}\right\}
$$

Hence, $F_{i j}$ is the net physical repulsive force between persons $i$ and $j, F_{i W}$ is the net physical repulsive force between persons $i$ and virtual wall element $\mathrm{W}, F_{p s i j}$ is the net psychological repulsive force between persons $i$ and $j$, $f_{i j}$ is the local physical repulsive force between persons $i$ and $j, f_{i W}$ is the local physical repulsive force between persons $i$ and $j$, and $f_{p s i j}$ is the local psychological repulsive force between persons $i$ and $j$.

$$
f_{*}=f_{*}^{n} n+f_{*}^{t} t
$$




$$
\begin{aligned}
& f_{*}^{n} n=(e+d)_{*}^{n} ; f_{*}^{t}(e+d)_{*}^{t} \\
& e_{*}^{n}=\left(e_{\text {pre }}+k \delta\right)_{*}^{n} ; e_{*}^{t}=\left(e_{\text {pre }}+k \delta\right)_{*}^{t} \\
& d_{*}^{n}=\left(c \frac{\delta}{\Delta t}\right)_{*}^{n} ; d_{*}^{t}=\left(c \frac{\delta}{\Delta t}\right)_{*}^{t}
\end{aligned}
$$

The superscripts $n$ and $t$ are the indicators for normal and tangential directions, $f$ is the inter-element force, $e$ is the component of the inter-element force due to the spring, $d$ is the component of the inter-element force due to the dashpot, $\delta$ is the relative displacement between contact elements, $\Delta t$ is the time marching step, $e$ is the component of the inter-element force due to the spring at the previous time step, $k$ and $c$ are the model parameters of the spring and dashpot, and the subscript * indicates any of the following subscripts: $i j, i W$ and $p s i j$. According to the contact condition between persons, the inter-element forces $f$ * given by Eq. (5) is modified as follow:

$$
\begin{aligned}
& e_{*}^{n}<0 \text { then } f_{*}=0 \\
& \left|e_{*}^{n}\right|>\mu e_{*}^{n} \text { then } f^{t}=\mu \bullet \operatorname{sign}\left[e_{*}^{n}, e_{*}^{t}\right]
\end{aligned}
$$

Where $\mu=0.577$ is the friction coefficient. Sign [A, B] represents the absolute value of $\mathrm{A}$ with the sign of $\mathrm{B}$.

\section{Results and Analysis}

This section shows the results obtained from the simulation that was ran by using Crowd Behavior Simulator for Disaster Evacuation (CBS-DE) model. Table 1 shows the population distribution for session three (3). Session three (3) was chosen as this session had the maximum capacity among all the convocation sessions in 2016. The population was calculated based on the number of seat provided as in Figure 1. While the number of visitor based on their gender was obtained by assuming the numbers of both male and female visitors were equal.

Table 1 Crowd distribution by gender for the maximum capacity convocation session

\begin{tabular}{cccc} 
Category & Gender & Type & $\begin{array}{c}\text { No. of } \\
\text { people }\end{array}$ \\
\hline $\mathbf{1}$ & Male & Adult & 1042 \\
$\mathbf{2}$ & Female & Adult & 1042 \\
& & Grand Total & $\mathbf{2 0 8 4}$ \\
\hline
\end{tabular}

There are two cases in this experiment where the average velocities used were different for both cases. They are:

i. Case 1: Average walking velocity of $1.16 \mathrm{~m} / \mathrm{s}$ was used for both male and female;

ii. Case 2: Average walking velocity of $1.25 \mathrm{~m} / \mathrm{s}$ was used for male and $1.12 \mathrm{~m} / \mathrm{s}$ was used for female.
Figure 7 shows the results of this experiment and the time taken for a complete evacuation for both cases were almost similar in which 145 seconds were taken for the evacuation of case 1 while 148 seconds for case 2 .

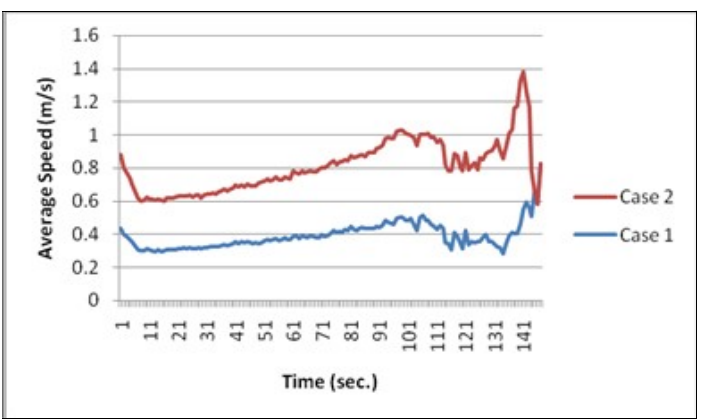

Fig. 7 This graph shows the average speed versus the time taken for the evacuation.

\section{Conclusion}

This study is a contribution to the control of emergency situation that is possible to happen at Dewan Sultan Ibrahim (DSI) not only during convocation days, but also at other times during large events. This study also helps in giving a better understanding about the flow of the evacuation process without even consuming so much time and also economical if compared to the classical way of evacuation training which is fire drill. However, the awareness of this kind of situation is vital and more information about the safety issues during emergency evacuation must be delivered to the public. Also, the design of any buildings must follow the standard of the design that has been enforced by the Uniform Building By-Laws (UBBL) 1984 of Malaysia. Hence, it can be concluded that all of the objectives have been achieved as for the first objective, the CBS-DE model is suitable to be used for DSI. Next, the data of simulation of human's speed during the evacuation process had also been applied to the experiment and from the obtained results, the time taken for the complete evacuation between the two cases were quite similar in which the evacuation of Case 1 took place for 145 seconds while 148 seconds were taken for Case 2 . Therefore, this software is one of the new methods that is highly recommended for a good evacuation planning process for a building in term of software other than fire drill without involving any volunteers.

\section{Acknowledgement}

This research is funded by the Ministry of Higher Education (MOHE) of Malaysia under Fundamental Research Grant Scheme (FRGS) Vot 1526.

\section{References}

[1] Abustan, S., 2013. Numerical Simulation of Evacuation Process Against Tsunami Disaster in Malaysia By Using Distinct-Element-Method Based Multi-Agent Model. 
[2] Duives, D. C., Daamen, W., \& Hoogendoorn, S. P. (2016). Continuum modelling of pedestrian flows Part 2: Sensitivity analysis featuring crowd movement phenomena. Physica A: Statistical Mechanics and Its Applications, 447, 36-48.

[3] Fahy, R.F., 2013. Overview of major studies on the evacuation of World Trade Center buildings 1 and 2 on 9/11. Fire Technol. 49, 643-655.

[4] Gotoh, H., Harada, E. \&Andoh, E., 2012. Simulation of pedestrian contra-flow by multi-agent DEM model with self-evasive action model. Safety Science, 50(2), pp.326-332.

[5] Gwynne, S. et al., 1999. A Review of the Methodologies Used in Evacuation Modelling. FIRE AND MATERIALS Fire Mater, 23, pp.383388.

[6] Ha, V.Q., 2012. Agent-based Modeling of Emergency Building Evacuation Agent-based Modeling of Emergency Building.

[7] Helbing, D. et al., 2005. Self-Organizing Pedestrian Movement. Environment and Planning B: Planning and Design, 28(3), pp.361-383.

[8] Kiyono, J., Miura, F. \&Takimoto, K., 1996. Simulation of Emergency Evacuation BehaviourDuring Disaster by Using Distinct Element Method. Doboku Gakkai Ronbunshu, 1996(537), pp.233-244.

[9] Kuligowski, 2005. Building and Fire Publications. Available at: http://fire.nist.gov/bfrlpubs/fire06/ art067.html.

[10] Lovreglio, R., Ronchi, E., Borri, D., 2014. The validation of evacuation simulation models through the analysis of behavioural uncertainty. Reliab. Eng. Syst. Saf. 131, 166-174. 